\section{When item recognition and visual search functions are similar*}

\author{
MARIANNE W. KRISTOFFERSON \\ Psychiatry Department, McMaster University, Hamilton, Ontario, Canada
}

The effect of prolonged practice upon item recognition performance was investigated under conditions of nested positive sets and complete response consistency. Nesting is defined by each positive set containing all the items contained in smaller positive sets. Response consistency is defined by each item in the stimulus set consistently requiring only a positive or only a negative response. A low error level was maintained. Twelve Ss worked with three positive set sizes in each of 36 sessions. Half the Ss worked with digit stimuli and half with pictures. The item recognition function (that function relating response latency and positive set size) was found to be negatively accelerated throughout the course of practice. The effect of positive set size decreased significantly $(p<.001)$ with practice, and set size effects were significantly $(p<.03)$ greater for positive response trials than for negative response trials. Kind of item had no effect on the set size effect. A theoretical framework consistent with these results is suggested. Results from the present study are compared with findings obtained previously from visual search studies. It is concluded that when the procedures in both tasks include response consistency, nested positive sets, and low error levels, the effects of prolonged practice upon the set size from item recognition and visual search are qualitatively very similar.

The number of items in the memory set (positive set size) has been shown to be an important variable in item recognition (Sternberg, 1966, 1969) and in visual search (Neisser, 1967). But set size seems to affect item recognition time differently than it does visual search time.

Visual search time increases as set size increases (the search function) early in practice, but the effect of set size diminishes with prolonged practice, reaching zero under some conditions (Neisser, Novick, \& Lazar, 1963; Wattenbarger, 1968) but stabilizing at a small value under others (Kristofferson, $1972 \mathrm{~b}$; Wattenbarger, 1968). The search function is negatively accelerated in the latter case. The critical difference between these two conditions seems to be error level. Visual search time is independent of set size after practice when error level is high, but not when error level is low.

Item recognition time also increases as set size increases (the item recognition function). The function is linear, and it has the same slope for both positive and negative responses. Set size typically has a relatively large effect upon item recognition time, amounting to $35-40$ msec per item (Sternberg, 1966). These findings have been confirmed, including the magnitude of the slope of the function

*This study was supported by a grant from the Medical Research Council of Canada, MA 3743. The author wishes to thank Linda Corsun and Carol Timusk for their assistance in data collection and analysis.
(Kristofferson, 1972a). Kristofferson showed further that only the intercept of the function changes with prolonged practice. The effect of set size is not influenced by practice. The function remains linear, and its slope does not change.

Thus, the effect of set size is profoundly different in search and in item recognition. In search, it is strongly influenced by practice, while in item recognition, it is not so affected.

There are many differences between Neisser's search task and Sternberg's item recognition task. Two of these differences are singled out here, and the item recognition task is modified in both ways to be more like the search task in order to determine whether the effect of practice upon the set size effect is altered. The two differences have to do with (1) the

Table 1

The Structure of Stimulus Sets for a Single Sention

\begin{tabular}{ccccc}
\hline & & \multicolumn{3}{c}{ Examples for Stimuli A-M } \\
\cline { 2 - 5 } Nesting & $\begin{array}{c}\text { Response } \\
\text { Consistency }\end{array}$ & $\begin{array}{c}\text { Positive } \\
\text { Set Size }\end{array}$ & $\begin{array}{c}\text { Positive } \\
\text { Set }\end{array}$ & $\begin{array}{c}\text { Negative } \\
\text { Set }\end{array}$ \\
\hline No & No & 1 & A & B-M \\
& & 2 & BC & A,D-M \\
No & Yes & 4 & DEFG & A-C, H-M \\
& & 1 & A & H-M \\
Yes & No & 2 & BC & H-M \\
& & 4 & DEFG & H-M \\
& & 1 & A & C-M \\
Yes & Yes & 2 & AB & E-M \\
& & 4 & ABCD & E-M \\
& & 1 & A & E-M \\
& & 2 & AB & E-M \\
\hline
\end{tabular}
experiment. present experiment will then be structure of the positive set, and (2) response consistency.

In Neisser's task, the positive sets are nested ${ }^{1}$, each set containing all of the items also contained in smaller sets. The item recognition task does not usually include this feature.

The search task also has complete response consistency in the sense that the items comprising the positive sets are never changed and they never appear as negative items. Sternberg's task, on the other hand, is characterized by response inconsistency: the same item is sometimes positive, sometimes negative, for each $S$.

The present experiment is designed to show the effect of prolonged practice upon the set size effect in item recognition when the procedure includes complete response consistency and nested positive sets. In order to extend the generality of the finding, two kinds of stimuli are used : digits and faces.

Examples of positive and negative stimulus set structures resulting from the possible combinations of nesting and response consistency are shown in Table 1. The final example illustrates the set structuring of the present

The results obtained from the compared with results obtained in a visual search experiment (Kristofferson, 1972b) when practice, error level, positive set structure, and response consistency were handled in the same way in the two studies.

\section{METHOD}

Apparatus and Stimulus Materials

A Scientific-Prototype three-field tachistoscope was used for presentation of the stimulus materials and the control of trial events. Two different sets of stimulus materials were prepared: one composed of Digits 0-9 and the other of photographs of faces. The digits were 
printed in black ink (Letraset, Franklin Gothic). The overall height of the digits was 1 in.; the visual angle subtended was approximately $1.25 \mathrm{deg}$. The set of pictures stimuli consisted of 10 black-and-white photographs of unfamiliar male faces. The photographs measured $2 \times$ 1-5/8 in., and the faces themselves were approximately $1 \mathrm{in}$. in height. Each picture and each digit was centered in the visual field.

\section{Design}

Twelve Ss were run individually in each of 36 daily sessions, excluding weekends. For half of the Ss, the pictures group, faces were used as the test items; for the remaining Ss, the numbers group, digits were used. Assignment of Ss to groups was random. There were three parts to each session, one for each of the three values of positive set size: $S=1, S=2$, and $S=4$. Each of the six possible orders of the three conditions were administered over each consecutive 6-day block, and the order within each 6-day block was determined randomly for each of the six pairs of Ss separately (one $S$ being a member of the numbers group and one a member of the pictures group). There were three successive blocks of 16 test trials for each of the set size conditions. Two practice trials preceded each block. There were 2-min breaks between blocks and 4-min breaks between conditions. Three $\mathrm{Ss}$ in each group were instructed to use their preferred hand for the positive response and the remaining three to use their nonpreferred hand. The other hand was used to respond to negative items.

\section{Assignment of Stimuli}

to Positive and Negative Sets

Separately for each of the six pairs of Ss, four of the numbers 0-9 were selected randomly without replacement from the total set of 10 to compose all of the positive sets for that pair of Ss. The assignment of digits and pictures to the three positive set size conditions was made for Session 1, as described below.

One number was drawn randomly without replacement from the four numbers composing the positive sets, and the digit or picture corresponding to that number was assigned to the $S=1$ condition. The $S=2$ condition was assigned the digit or picture used in the $S=1$ condition, plus the digit or picture which corresponded to a second randomly drawn number from the total positive set. The $S=4$ condition had assigned to it all four of the digits or pictures corresponding to the numbers composing the positive sets. For all subsequent sessions, the total set of four stimuli making up the positive sets was the same as in Session 1. However, to equalize the frequency of occurrence of each of the four positive stimuli within each positive set size condition for each $S$, over successive 12 sessions, each member of the positive set was assigned randomly 3 times to the $S=1$ condition, 6 times to the $S=2$ condition, and 12 times to the $S=4$ condition. Thus, over the course of the experiment, each of the four digits composing a given S's positive sets appeared equally often (216 times) as a member of each of the three positive set size conditions. The negative set, in all sessions and in all conditions, was composed of the six digits or six pictures remaining after the positive set had been selected. Thus, for each $\mathbf{S}$ throughout the experiment, each item always required only a positive or only a negative response.

\section{Trial Events}

The sequence of events for each trial was as follows: A white card with a centered black dot, which served both as a warning signal and a fixation aid, was displayed for $1.50 \mathrm{sec}$. It was immediately replaced by the appropriate test stimulus (a digit or a face), which was displayed for 440 msec. A blank white card was then displayed for the remainder of the 15-sec intertrial interval. Following the presentation of the test stimulus, the $\mathbf{S}$ responded by pressing one of the two telegraph keys to indicate whether the test stimulus was or was not a member of the previously defined positive set. The time from the onset of the test stimulus to the S's response was recorded with an accuracy of \pm 1 msec. Auditory feedback informed the $S$ as to the correctness of his response.

\section{Test Stimulus Sequence}

For each of the set size conditions, $50 \%$ of the trials required a positive response. With that restriction, for each day and each condition, a different random ordering of positive and negative trials was used. When the positive set contained more than one digit or picture, each item in the set was presented randomly but equally of ten. For all conditions and each day, the six stimuli composing the negative set were presented randomly but equally often.

\section{Subjects and Instructions}

The Ss were male volunteers from the student body of McMaster University and were paid \$2 per session. None of the Ss had had any previous exposure to the experimental task.

Before each set size condition and prior to each block, the $\mathbf{S}$ was shown the composition of the positive set. For example, a right-handed $S$ in the pictures group who was instructed to make positive responses with his preferred hand would be shown in sequence the faces for which the right-hand key was correct, and further instructed that the other key was correct for all other faces.

Mean response latencies and mean percent of errors over all three conditions were calculated and posted daily for the Ss to see. The Ss were encouraged to respond rapidly but to maintain their errors below $2 \%$.

\section{RESULTS}

All correct responses, excepting practice trials, were used for analysis. Mean response time is shown plotted against positive set size for each of the six successive 6-day blocks and for negative and positive trials separately for the numbers group in Fig. 1 and for the pictures group in Fig. 2. Best-fitting straight lines were determined for each of these 24 sets of data, and the percent of variance accounted for by linear regression in each case is presented in Table 1.

In view of the fact that linear regression is typically found to account for approximately $99 \%$ of the variance (Sternberg, 1966, 1967; Kristofferson, 1972a), inspection of Table 1 leads to the conclusion that these data are not well described by linear functions, especially after the first 6-day block. It is also apparent from visual inspection of Figs. 1 and 2 that the functions relating set size and response latency are negatively accelerated rather than linear. For this reason, an analysis in terms of slope cannot be done, and instead, difference scores were calculated from the average response latencies to the three positive set sizes to provide estimates of the average increase in response latency when the positive set size was increased from 1 to 2 and from 1 to 4.

For each S separately, differences between the mean response latency when the positive set was composed of two items and when it contained only one item $\left(\overline{\mathrm{RL}}_{\mathrm{S}=2}-\overline{\mathrm{RL}}_{\mathrm{S}=1}\right)$ were calculated for each successive 6-day block and for positive and negative trials separately. These difference scores, $\mathrm{D}_{2}$, provide one way of estimating the average amount by which response latency is increased by the addition of one member to a positive set size of one. In a similar way, for each $S$, a set of estimates of the average amount by which response latency was increased from one item to four items, $D_{4}$, was calculated by determining the differences in mean 
response latency when the positive set was composed of four items and when it was a single item, and dividing this difference by 3 , the number of added items: $\left(\overline{\mathrm{RL}}_{\mathrm{S}=4}-\overline{\mathrm{RL}}_{\mathrm{S}=1}\right) / 3$. For each $\mathrm{S}$, then, $12 \quad \mathrm{D}_{2}$ estimates were calculated, one for each of the six successive 6-day blocks of trials, using only response latencies to trials requiring a positive response, and one for each of the six successive 6-day blocks of trials, using only response latencies to trials requiring a negative response. Similarly, for each $\mathrm{S}, 12 \mathrm{D}_{4}$

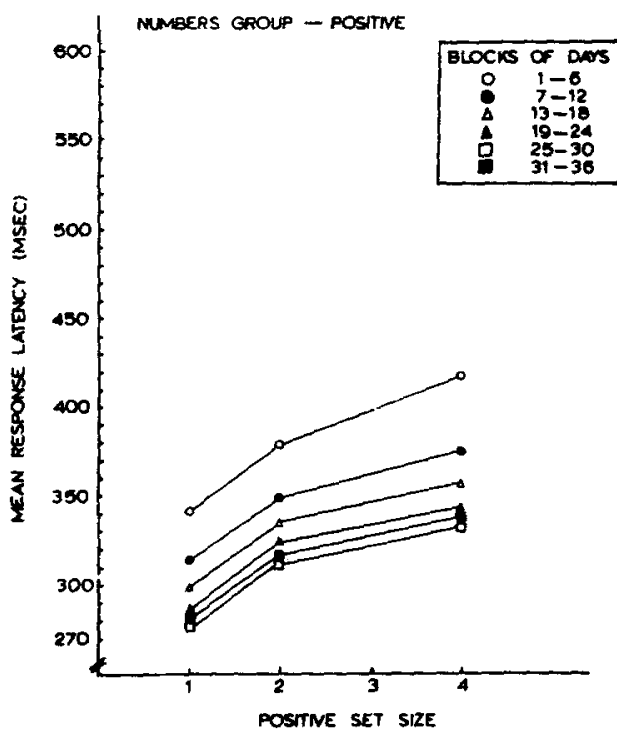

Fig. 1. Mean response latency, calculated separately for trials requiring a positive and trials requiring a negative response, shown as a function of set size for each successive 6 -day block, where numbers were the stimuli.

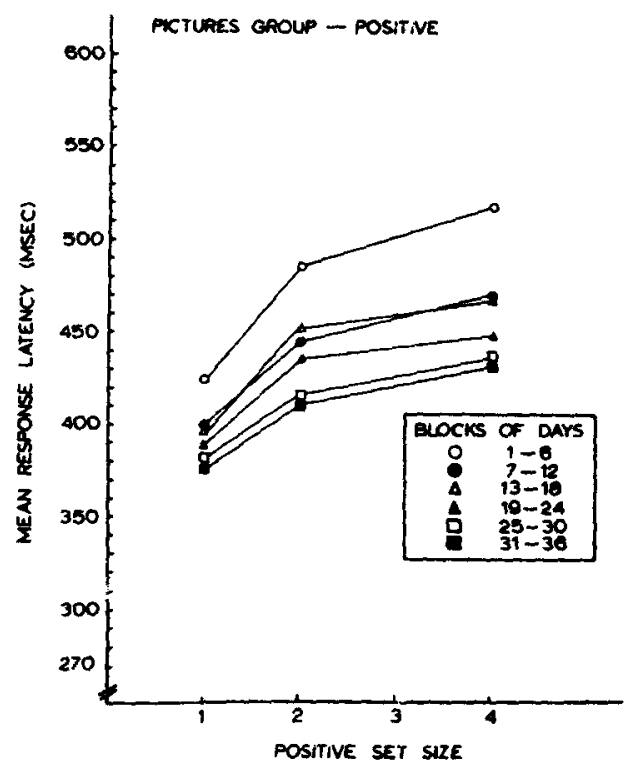

Fig. 2. Mean response latency, calculated separately for trials requiring a positive and trials requiring a negative response, shown as a function of set size for each successive 6 -day block, where pictures were the stimuli.
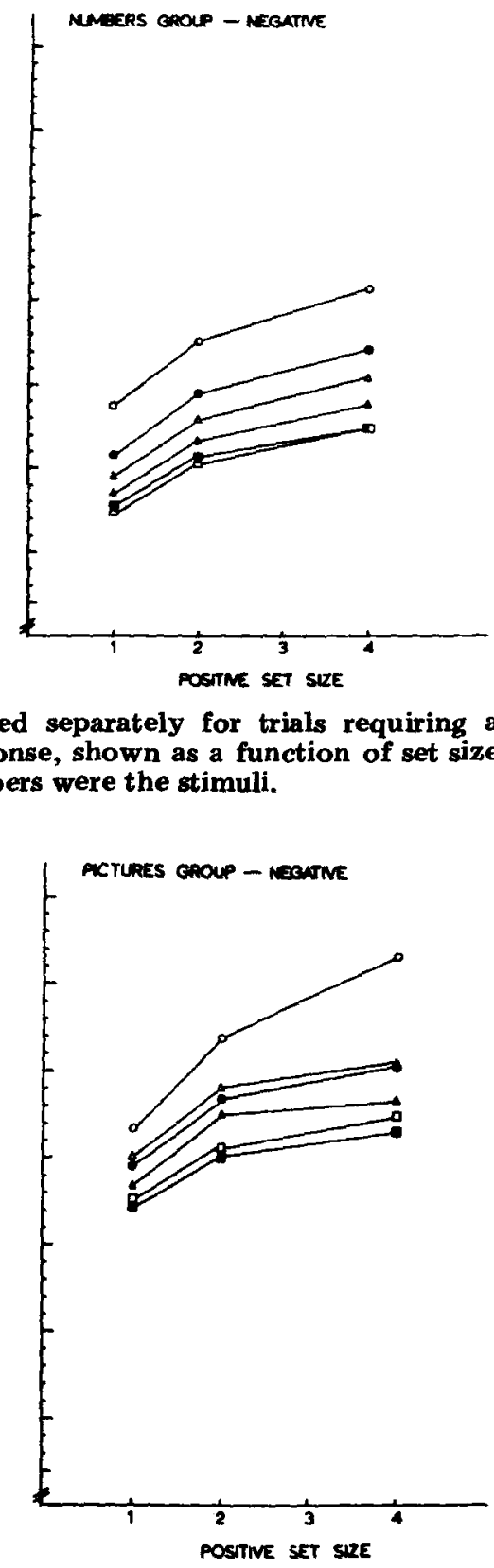

Table 2

Percent $V$ ariance Accounted for by Linear Rerreasion

\begin{tabular}{lcccc} 
& \multicolumn{2}{c}{ Numbers } & \multicolumn{2}{c}{ Pictures } \\
\cline { 2 - 4 } $\begin{array}{c}\text { Day } \\
\text { Blocks }\end{array}$ & $\begin{array}{c}\text { Positive } \\
\text { Trials }\end{array}$ & $\begin{array}{c}\text { Negative } \\
\text { Trials }\end{array}$ & $\begin{array}{c}\text { Positive } \\
\text { Trials }\end{array}$ & $\begin{array}{c}\text { Negative } \\
\text { Trials }\end{array}$ \\
\hline $1-6$ & 96.6 & 95.1 & 87.3 & 94.3 \\
$7-12$ & 91.6 & 93.4 & 88.1 & 85.3 \\
$13-18$ & 89.8 & 91.2 & 78.1 & 81.5 \\
$19-24$ & 85.6 & 90.6 & 78.0 & 69.2 \\
$25-30$ & 89.5 & 87.9 & 89.2 & 87.7 \\
$31-36$ & $\mathbf{8 1 . 5}$ & 87.6 & 89.8 & 87.6 \\
\hline
\end{tabular}

The difference scores were analyzed by a four-factor mixed design ANOVA with repeated measures on three factors, where the between factor was groups (pictures or digits) and the within factors were (1) blocks of days, (2) trials requiring positive or negative responses, and (3) $D_{2}$ vs $D_{1}$.

Overall, there was no significant difference between the performance of the two groups $[F(1,10)<1]$ and none of the interactions were significant at the .05 level or less.

The main effect of $\mathrm{D}_{2}$ vs $\mathrm{D}_{4}$, method of estimating the average time added to the comparison stage by the addition of one item to a positive set of one, was significant $[F(1,10)=$ $90.11, p<.00011$. Lower estimates were obtained from $D_{4}$ difference scores. In Fig. 3, for the two groups combined, $\mathrm{D}_{2}$ and $\mathrm{D}_{4}$ are each shown plotted against blocks of days separately for negative and positive responses. This significant effect again demonstrates the negative acceleration of the function relating response latency and positive set size. And, together with the absence of any significant interactions, it also implies that the functions relating these two variables were similar for the two groups.

There was a significant main effect of positive-negative $[F(1,10)=5.87$, $\mathrm{p}<.03$ ], with $\mathrm{D}_{2}$ and $\mathrm{D}_{4}$ calculated from positive trials being greater than those from negative trials. The set size effect was found to be greater for trials requiring a positive response. Finally, the overall effect of days was significant $[F(5,50)=7.06$, p $<.0001]$. Difference scores, $D_{2}$ and $\mathrm{D}_{4}$, decreased over blocks ofdays; or, in other words, the set size effect decreased with practice. No $S$ in either group made errors on more than $3.7 \%$ of the total trials. For the numbers group, the average percent errors for each of the three positive set sizes was $.9 \%, 1.8 \%$, and $2.5 \%$ for positive set sizes of 1,2 , and 4 , respectively. The analogous figures for the pictures group were $1.7 \%, 2.3 \%$, and $2.6 \%$.

\section{DISCUSSION}

The results show quite clearly that throughout the course of practice, the effect of set size on response latency 


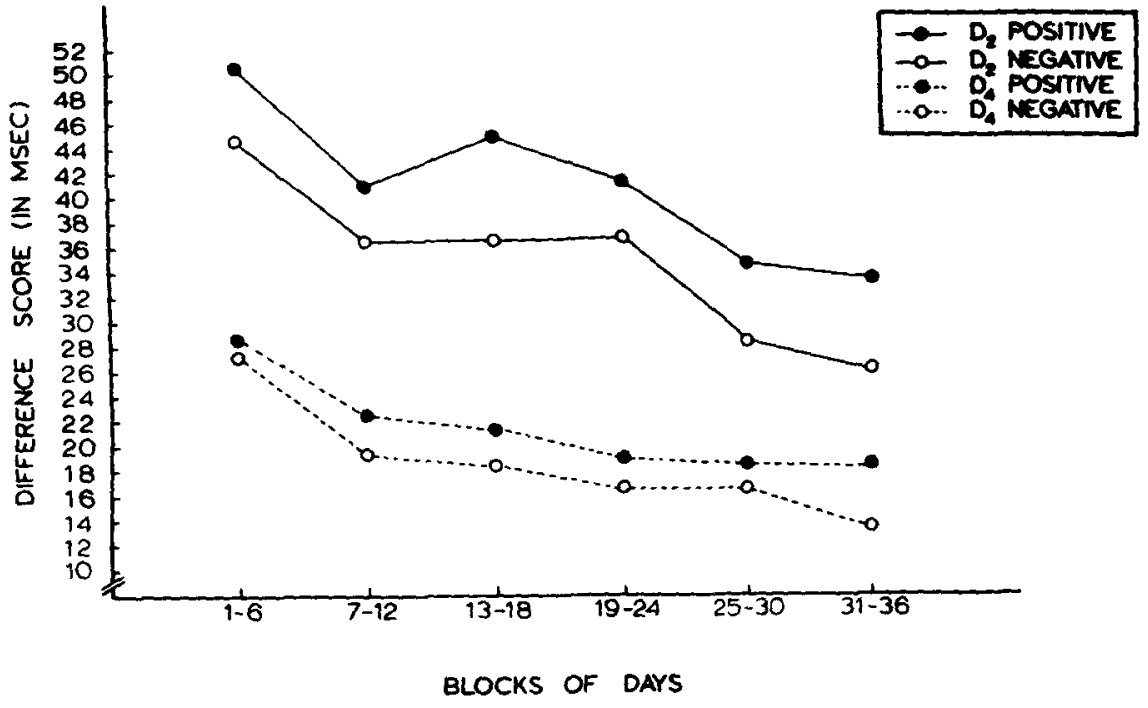

Fig. 3. Difference scores, $D_{2}$ and $D_{4}$, where each is calculated separately from positive and from negative trials, shown as a function of successive 6-day blocks.

does not differ significantly for either negative or positive trials when digits or faces are used as the stimulus item sets. These results lead to the conclusion that under the conditions of this experiment the effect of set size is independent of the kind of item. In more theoretical terms, the results are consistent with the conclusion that neither the nature nor the rate of the process by which comparisons between memory item representations and the display item representation are made is dependent upon the kind of item.

The performance of the two groups is not without differences, however. As shown in Fig. 4, the overall mean response latency averaged over the three positive set size conditions is consistently greater for the pictures group, and this difference, averaging approximately $100 \mathrm{msec}$, is quite stable throughout practice. This suggests that the pictures stimuli were more "difficult" in some respect, but that whatever additional processing was required for these stimuli took place in some stage other than the comparison stage. It also suggests that prior to the comparison stage, the stimulus representation is transformed and coded into the same mode for the two different sets of stimuli. And this coding of the test stimulus representation appears to be established quite early in practice, at least under the conditions of this experiment, since even the average comparison rate over the first 6-day block does not differentiate the two groups.

Under the conditions of this experiment, the item recognition function was found to be negatively accelerated. The effect of set size significantly decreased with practice forboth negative and positive trials. And, throughout the course of practice, the effect of set size was significantly greater for positive than for negative trials. These results contrast markedly with the findings obtained in the Kristofferson (1972a) study, which differed from thepresent experiment only in that response inconsistency and nonnested positive sets were used. In that experiment, a linear item recognition function was found. The effect of set size was stable over the course of practice; and the

Fig. 4. Mean response latency as a function of practice. This relationship is shown separately for pictures and numbers stimuli, and in each case separately for positive and negative trials. response consistency alone or to the joint effects of response consistency and nested positive sets. To determine whether only one or both of these conditions is necessary to produce these changes, the nesting variable and the response consistency variable must be independently manipulated within the same experiment.

It is doubtful that the negatively accelerated item recognition functions found in the present study can be attributed to the fact that errors, though low overall, increased with set size. In the present study, mean errors

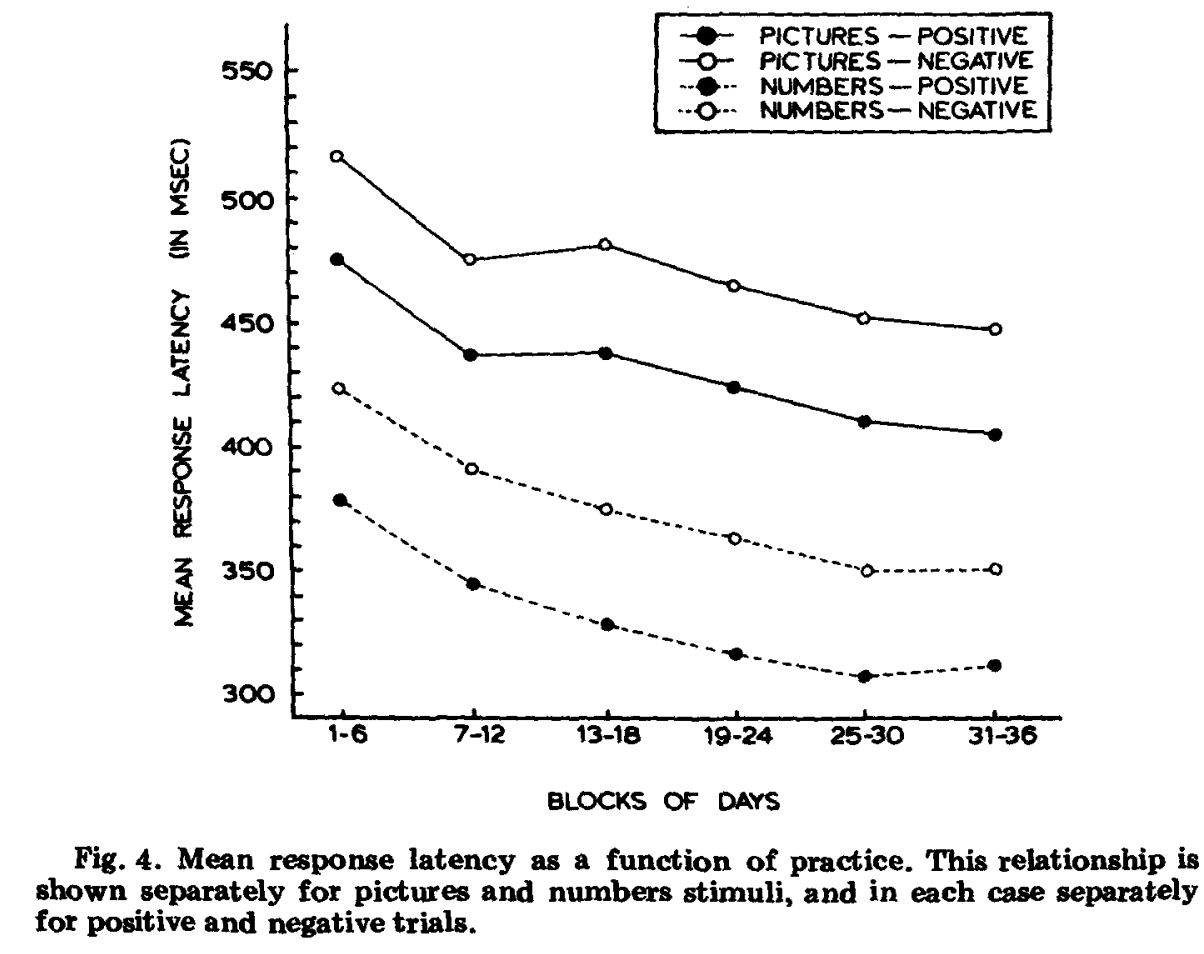

effect of set size was the same for positive and for negative trials.

A set of general conclusions from these data are: (1) response consistency either alone or in combination with constant and nested positive sets has powerful effects on the nature and rate of the comparison process; (2) some of these effects are amplified with prolonged practice; and (3) the effects are similar for the two types of stimulus sets used.

Burrows and Murdock (1969) have studied the effects of practice on item recognition performance with constant and nested positive sets, but with response inconsistency. ${ }^{2}$ They report that response latency increased as a positive function of set size and that practice did not attenuate the effect of set size. Their findings suggest that the departures from the usual item recognition results found in the present study are not attributable to the use of constant and nested positive sets alone, but are due either to 
for the numbers group were $.7 \%, 1.8 \%$, and $2.5 \%$ for positive set sizes of 1,2 , and 4, respectively. In the Kristofferson (1972a) study, which also used digits, the comparable error rates were $.7 \%, 1.6 \%$, and $2.5 \%$. In both studies, error rate increased with set size, and the percent of errors for each set size was almost identical in the two studies. One would expect the difference in error levels to exert the same influence in both studies.

of the results from thepresent experiment, the one finding which is hardest to reconcile with Stermberg's (1966, 1969) exhaustive serial comparison model of memory scanning is that the set size effect (the comparison rate) is significantly greater for positive trials. Equivalent comparison rates for positive and for negative trials are required to support a hypothesis of exhaustive scanning of the positive set prior to responding. Nor are these data consistent with a hypothesis of a self-terminating scanning of the positive set, since support of that hypothesis requires that the comparison rate obtained from positive trials be one-half of the rate obtained from negative trials, under the conditions of this experiment. How might this finding be accounted for? One possibility is that the comparison of the test item with items in the positive sets is not a series of unitary discrete events where the number of events has as its maximum the number of items compasing the positive set. But rather that, at least under the conditions of this experiment (which provides specific and prolonged practice on the items which consistently compose the positive sets and which also ties each item to only a positive or only a negative response over the course of the experiment), features which uniquely define each positive set are organized into a hierarchy of tests, and these tests are made in sequence only until enough information is obtained to determine whether the test item is or is not a member of the positive set. On the average, fewer tests would be required to determine that a test item was not a member of the positive set than to determine that an item was a member of the positive set.

By conceptualizing the comparison process in this way, it is also possible to account for (1) the negatively accelerated function that was found to describe the response-latency/set-size relationship, and (2) the finding that $D_{2}$ and $D_{4}$ decreased with practice. If a hierarchy of such feature tests is devised for any given positive set of items, while the number of tests would be expected to increase as some function of the number of items composing the positive set, it seems

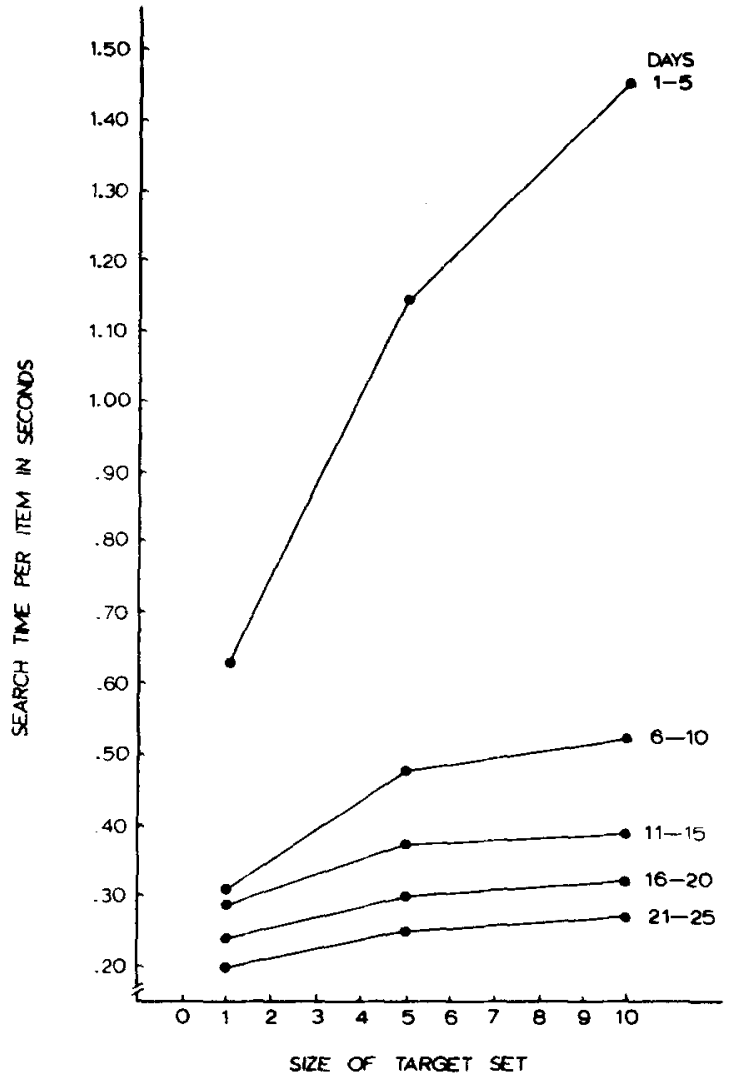

Fig. 5. Search time per item shown as a function of target set size for each successive 5-day block separately.

reasonable to speculate that the number of tests added decreases as the size of the positive set increases. Further, with practice, a set of such feature tests might be expected to become more efficient.

How do the results from the present study compare qualitatively with the results obtained from the search task when practice, error level, positive set structure, and response consistency are handled in the same way in the two tasks?

In the main search experiment reported by Kristofferson (1972b), data were collected on target set sizes of 1,5 , and 10 on each of 25 days from eight Ss. These Ss performed with a low error rate throughout the experiment. Slopes were determined by calculating the equation for the least squares linear function relating response latencies and target positions for each $\mathbf{S}$, each set size condition, and each day, separately. These slope values, which are interpreted as representing the time required to scan over each nontarget item independently of response factors, were combined and averaged over the eight Ss and over successive 5-day blocks for each set size, separately. These data, which are replotted from the original study, are presented in
Fig. 5, where the function relating set size and slope (search time) in seconds is shown for different levels of practice. For the data obtained from the item recognition experiment, the same kind of relationship, comparison time as a function of set size at different levels of practice is shown in Figs, 1 and 2.

The theoretically important features of the data are quite similar for the three sets of data. Throughout the course of practice, the search functions and the item recognition functions are negatively accelerated. The effect of set size decreases with practice, but even after prolonged practice, a significant set size effect is present. Finally, for each of the positive set sizes, search time (response latency) decreases over the course of practice.

It was found in thepresent experiment that the set size effect was significantly smaller for items requiring a negative response than for items requiring a positive response (Fig. 3). As was pointed out earlier, this finding is inconsistent with either an exhaustive or a self-terminating scan of the positive set. This finding seems consistent with certain results that have been obtained in visual search (Neisser, 1963). In Neisser's 
study, Ss sometimes had the task of searching for the presence of an item, or items, and they sometimes had the task of searching for the absence of an item, or items. When searching for presence, search times reflect the speed with which the Ss scan through list items when the list items do not match the items in memory (negative comparison). When the task is searching for absence, search times reflect two types of comparisons with the memory set: positive and negative. Neisser found the slope of the search function to be significantly greater when searching for absence than when searching for presence. These findings are consistent with the results from the present experiment in that they show that the search function is greater for positive and negative trials combined than for negative trials alone.

In summary, with response consistency, nested sets, and low error levels, the effects of prolonged practice upon the set size effect in item recognition and visual search are qualitatively very similar.
If it is true that the joint effects of these variables are responsible for the conflicting conclusions produced from search and item recognition experiments, certain implications follow. For example, these results lead to the prediction that serial processing should be obtained in visual search when (1) errors are low and (2) response inconsistency prevails. When these two conditions are met, it should be the case that the search function is linear and that its slope is not affected by practice. REFERENCES
BURROWS, D., \& MURDOCK, B. B., JR.
Effects of extended practice on
high-speed scanning. Journal of
Experimental Psychology, 1969, B2. 231-237.

KRISTOFFERSON, $M$. W. Effects of practice on character-classification performance. Canadian Journal of Psychology, 1972a, 26, 54-60.

KRISTOFFERSON, $M$, Types and frequency of errors in visual search. Perception \& Pychophysics, 1972b, 11 , 325-328.

NEISSER, U. Decision-time without reaction-time: Experiments in visual s canning. American Journal of Psychology, 1963, 76, 376-385.
NEISSER, U. Cognitive psychology. New York: A ppleton-Century-Crofts, 1967.

NEISSER, $U$ NOVICK, $R$ \& IAZAR, Searching for ten targets simultaneously. Perceptual \& Motor Skills, 1963, 17, 955-961.

STERNBERG, S. High-speed scanning in human momory. Science, 1966, 153, 652-654.

STERNBERG, S. Memory-scanning: Mental processes revealed by reaction-time experiments. American Scientist, 1969. 57, 421-457.

WATTENBARGER, B. $L$. Speed and accuracy set in visual serach performance. Paper presented at the meeting of the Midwestern Psychological Association, Chicago, 1968.

\section{NOTES}

1. Actually, there is not complete nesting in the search experiments, since in the Neisser et al (1963), Wattenbarger (1968), and Kristofferson (1972b) studies the following four target sets were used: (1) "K," (2) "AFKU9," (3) "HMPZ4," and (4) "AFKU 9HMPZ4." Target Set 3 does not include the character in (1), yet performance on (2) and (3) did not differ after a small amount of practice.

2. Personal communication, D. Burrows, July 5, 1971.

(Accepted for publication June 19, 1972.) 